\title{
On Bilateral Generating Functions of Konhauser Biorthogonal Polynomials
}

\author{
K.P. Samanta ${ }^{1, *}$, B. Samanta ${ }^{2}$ \\ ${ }^{1}$ Department of Mathematics, Indian Institute of Engineering Science and Technology, Shibpur, India \\ ${ }^{2}$ Department of Mathematics, Shibpur D.B. Institution(College), India
}

Copyright (C) 2015 Horizon Research Publishing All rights reserved.

\begin{abstract}
In this article, we have obtained some novel results on bilateral generating functions of the polynomials, $Y_{n+r}^{\alpha-n k}(x ; k)$, a modified form of Konhauser biorthogonal polynomials, $Y_{n}^{\alpha}(x ; k)$ by group-theoretic method. As special cases, we obtain the corresponding results on Laguerre polynomials, $L_{n}^{(\alpha)}(x)$. Some applications of our results are also discussed.
\end{abstract}

Keywords Laguerre Polynomials, Biorthogonal Polynomials, Generating Functions

AMS-2010 Subject Classification Code: 33C 45, 33C 47

\section{Introduction}

In 1965[1], Konhauser extended the notion of a particular pair of biorthogonal polynomial sets as introduced by Spencer and Fano [3] and established general properties of biorthogonal sets. In [2], Konhauser also introduced two sets of polynomials $\left\{Y_{n}^{\alpha}(x ; k)\right\}$ and $\left\{Z_{n}^{\alpha}(x ; k)\right\}$, which are biorthogonal with respect to the weight function $x^{\alpha} e^{-x}$ over the interval $(0, \infty), \alpha>-1, k$ is a positive integer. These polynomials satisfy the following condition:

$$
\int_{0}^{\infty} x^{\alpha} \exp (-x) Y_{i}^{\alpha}(x ; k) Z_{j}^{\alpha}(x ; k) d x\left\{\begin{array}{cc}
=0, & i \neq j, \\
\neq 0, & i=j ;
\end{array} i, j\right.
$$

For $k=1$, these polynomials reduce to the generalized Laguerre polynomials, $L_{n}^{\alpha}(x)$. For previous works on these polynomials one can see the works [7-14]. In the present paper we are interested only on $Y_{n}^{\alpha}(x ; k)$. In [7], Carlitz gave an explicit representation for the polynomials $Y_{n}^{\alpha}(x ; k)$ in the following form:

$$
Y_{n}^{\alpha}(x ; k)=\frac{1}{n !} \sum_{i=0}^{n} \frac{x^{i}}{i !} \sum_{j=0}^{i}(-1)^{j}\left(\begin{array}{l}
i \\
j
\end{array}\right)\left(\frac{j+\alpha+1}{k}\right)_{n},
$$

where $(a)_{n}$ is the pochhammer symbol defined by

$$
(a)_{n}=\frac{\Gamma(a+n)}{\Gamma(a)}=\left\{\begin{array}{cc}
1, & \text { if } n=0, \quad a \neq 0 \\
a(a+1) \ldots(a+n-1), & \forall n \in\{1,2,3 \ldots\} .
\end{array}\right.
$$

The aim at presenting this paper is to obtain some novel theorems on bilateral generating relations for the polynomials, $Y_{n+r}^{\alpha-n k}(x ; k)$, a modified form of Konhauser biorthogonal polynomials, $Y_{n}^{\alpha}(x ; k)$ by the group-theoretic method. As special cases, we obtain the corresponding results on Laguerre polynomials, $L_{n}^{(\alpha)}(x)$. The main results of our investigation are stated in the form of the following theorems:

Theorem 1. If there exists a unilateral generating relation of the form

$$
G(x, w)=\sum_{n=0}^{\infty} a_{n} Y_{n+r}^{\alpha-n k}(\mathrm{x} ; \mathrm{k}) w^{n}
$$

then

$$
\begin{gathered}
(1+k t)^{\frac{(1+\alpha-k)}{k}} \exp \left\{x-x(1+k t)^{\frac{1}{k}}\right\} G\left(x(1+k t)^{\frac{1}{k}}, \frac{v t}{1+k t}\right) \\
=\sum_{n=0}^{\infty} Y_{n+r}^{\alpha-n k}(\mathrm{x} ; \mathrm{k}) \sigma_{n}(v) t^{n},
\end{gathered}
$$

where

$$
\sigma_{n}(v)=\sum_{p=0}^{n} a_{p} k^{n-p}\left(\begin{array}{c}
n+r \\
p+r
\end{array}\right) v^{p} .
$$

Theorem 2. If there exists a unilateral generating relation of the form

$$
G(x, w)=\sum_{n=0}^{\infty} a_{n} Y_{n+r}^{\alpha}(\mathrm{x} ; \mathrm{k}) w^{n}
$$

then

$$
\begin{aligned}
& (1+k t)^{\frac{(1+\alpha-k)}{k}} \exp \left\{x-x(1+k t)^{\frac{1}{k}}\right\} G\left(x(1+k t)^{\frac{1}{k}}, v t\right) \\
& =\sum_{n=0}^{\infty} \sigma_{n}(x, v) t^{n},
\end{aligned}
$$

where

$$
\sigma_{n}(x, v)=\sum_{p=0}^{n} a_{p} k^{n-p}\left(\begin{array}{c}
n+r \\
p+r
\end{array}\right) Y_{n+r}^{\alpha-n k+p k}(\mathrm{x} ; \mathrm{k}) v^{p} .
$$

Finally, we like to point it out that some applications of our theorems are also given in the paper. 


\section{Operator and Extended Form of the Group}

At first, we seek a linear partial differential operator $R$ of the form:

$$
R=A_{1} \frac{\partial}{\partial x}+A_{2} \frac{\partial}{\partial y}+A_{0}
$$

where each $A_{i}(i=0,1,2,3)$ is a function of $x$ and $y$ which is independent of $n$ such that

$$
R\left(Y_{n+r}^{\alpha-n k}(\mathrm{x} ; \mathrm{k}) y^{n}\right)=\Omega_{n} Y_{n+r+1}^{\alpha-n k-k}(\mathrm{x} ; \mathrm{k}) y^{n+1},
$$

where $\Omega_{n}$ is a function of $n$ and is independent of $x$ and $y$.

Using (5) and with the help of the differential recurrence relation:

$$
\begin{gathered}
x \frac{d}{d x}\left[Y_{n+r}^{\alpha-n k}(x ; k)\right] \\
=k(n+r+1) Y_{n+r+1}^{\alpha-n k-k}(x ; k)+ \\
+(x+k+n k-\alpha-1) Y_{n+r}^{\alpha-n k}(x ; k)
\end{gathered}
$$

we easily obtain the following linear partial differential operator:

$$
R=x y \frac{\partial}{\partial x}-k y^{2} \frac{\partial}{\partial y}-(x+k-\alpha-1) y
$$

such that

$R\left(Y_{n+r}^{\alpha-n k}(\mathrm{x} ; \mathrm{k}) y^{n}\right)=k(n+r+1) Y_{n+r+1}^{\alpha-n k-k}(\mathrm{x} ; \mathrm{k}) y^{n+1}$. by

The extended form of the group generated by $R$ is given

$$
\begin{gathered}
e^{w R} f(x, y) \\
=(1+k w y)^{\frac{1+\alpha-k}{k}} \exp \left\{x-x(1+k w y)^{\frac{1}{k}}\right\} \\
\times f\left(x(1+k w y)^{\frac{1}{k}}, \frac{y}{1+k w y}\right),
\end{gathered}
$$

where $f(x, y)$ is an arbitrary function and $w$ is an arbitrary constant.

\section{Derivation of Generating Function}

Now writing $f(x, y)=Y_{n+r}^{\alpha-n k}(x ; k) y^{n}$ in (8), we get $e^{w R}\left(Y_{n+r}^{\alpha-n k}(x ; k) y^{n}\right)$

$=(1+k w y)^{\frac{1+\alpha-n k-k}{k}} \exp \left\{x-x(1+k w y)^{\frac{1}{k}}\right\}$

$\times Y_{n+r}^{\alpha-n k}\left(x(1+k w y)^{\frac{1}{k}} ; k\right) y^{n}$.

Again, using (7), we obtain

$$
\begin{gathered}
e^{w R}\left(Y_{n+r}^{\alpha-n k}(x ; k) y^{n}\right)= \\
\sum_{m=0}^{\infty} k^{m} \frac{w^{m}}{m !}(n+r+1)_{m} Y_{n+r+m}^{\alpha-n k-m k}(x ; k) y^{n+m} .
\end{gathered}
$$

Equating (9) and (10) and then substituting $w y=t$, we get

$$
\begin{gathered}
(1+k t)^{\frac{1+\alpha-n k-k}{k}} \exp \left\{x-x(1+k t)^{\frac{1}{k}}\right\} Y_{n+r}^{\alpha-n k}\left(x(1+k t)^{\frac{1}{k}} ; k\right) \\
=\sum_{m=0}^{\infty} k^{m}\left(\begin{array}{c}
n+r+m \\
m
\end{array}\right) Y_{n+r+m}^{\alpha-n k-m k}(x ; k) t^{m},
\end{gathered}
$$

which does not seem to have appeared in the earlier works.

Corollary 1: Replacing $\alpha$ by $\alpha+n k$ in (11), we get

$$
\begin{gathered}
(1+k t)^{\frac{\alpha+1-k}{k}} \exp \left(x-x(1+k t)^{\frac{1}{k}}\right) Y_{n+r}^{\alpha}\left(x(1+k t)^{\frac{1}{k}} ; k\right) \\
=\sum_{m=0}^{\infty} k^{m}\left(\begin{array}{c}
n+r+m \\
m
\end{array}\right) Y_{n+r+m}^{\alpha-m k}(x ; k) t^{m} .
\end{gathered}
$$

Corollary 2: Again, putting $n=0$ in (12), we get

$$
\begin{gathered}
(1+k t)^{\frac{\alpha+1-k}{k}} \exp \left(x-x(1+k t)^{\frac{1}{k}}\right) Y_{r}^{\alpha}\left(x(1+k t)^{\frac{1}{k} ; k}\right) \\
=\sum_{m=0}^{\infty} k^{m}\left(\begin{array}{c}
r+m \\
m
\end{array}\right) Y_{r+m}^{\alpha-m k}(x ; k) t^{m},
\end{gathered}
$$

which is found derived in $[11,17]$.

Corollary 3: Putting $r=0$ in (13), we get

$$
\begin{gathered}
(1+k t)^{\frac{\alpha+1-k}{k}} \exp \left(x-x(1+k t)^{\frac{1}{k}}\right)= \\
\sum_{m=0}^{\infty} k^{m} Y_{m}^{\alpha-m k}(x ; k) t^{m},
\end{gathered}
$$

which is found derived in $[11,13,17]$ by different methods.

Special case 1:If we put $k=1$, then $Y_{n}^{\alpha}(x ; k)$ reduces to the generalized Laguerre polynomials, $L_{n}^{\alpha}(x)$. Thus Putting $k=1$ in (11) - (14), we get the following generating relation on Laguerre polynomials:

$$
\begin{aligned}
&(1+t)^{\alpha-n} \exp (-x t) L_{n+r}^{\alpha-n}(x(1+t)) \\
&=\sum_{m=0}^{\infty}\left(\begin{array}{c}
n+r+m \\
m
\end{array}\right) L_{n+r+m}^{\alpha-n-m}(x) t^{m} . \\
&(1+t)^{\alpha} \exp (-x t) L_{n+r}^{\alpha}(x(1+t)) \\
&=\sum_{m=0}^{\infty}\left(\begin{array}{c}
n+r+m \\
m
\end{array}\right) L_{n+r+m}^{\alpha-m}(x) t^{m} . \\
&(1+t)^{\alpha} \exp (-x t) L_{r}^{\alpha}(x(1+t)) \\
&=\sum_{m=0}^{\infty}\left(\begin{array}{c}
r+m \\
m
\end{array}\right) L_{m+r}^{\alpha-m}(x) t^{m},
\end{aligned}
$$

which is found derived in $[15,16,18,19,20,22]$.

$$
(1+t)^{\alpha} \exp (-x t)=\sum_{m=0}^{\infty} L_{m}^{\alpha-m}(x) t^{m},
$$

which is found derived in [23].

Special case 2: Using the relation $L_{n}^{\alpha-n}(x)=\frac{(-x)^{n}}{n !} C_{n}(\alpha ; x),[6$, pp.68-69] and from the above generating relations we get the following generating relations on Charlie rpolynomials [4, p. 226]:

$$
\begin{gathered}
\left(1-\frac{y}{x}\right)^{\alpha+r} \exp (y) C_{n+r}(\alpha+r ; x-y) \\
=\sum_{m=0}^{\infty} \frac{y^{m}}{m !} C_{n+r+m}(\alpha+r ; x)
\end{gathered}
$$




$$
\begin{gathered}
\left(1-\frac{y}{x}\right)^{\alpha} \exp (y) C_{n}(\alpha ; x-y) \\
=\sum_{m=0}^{\infty} \frac{y^{m}}{m !} C_{n+m}(\alpha ; x)
\end{gathered}
$$

which is found derived in $[6, \mathrm{pp} .70]$ and equivalent to Doetsch's formula given in [5, pp. 88(26)].

$$
\left(1-\frac{y}{x}\right)^{\alpha} \exp (y)=\sum_{m=0}^{\infty} \frac{y^{m}}{m !} C_{m}(\alpha ; x) .
$$

Now we proceed to prove the Theorem 1 .

\section{Proof of Theorem 1}

Let us consider the generating relation of the form:

$$
G(x, w)=\sum_{n=0}^{\infty} a_{n} Y_{n+r}^{\alpha-n k}(x ; k) w^{n} .
$$

Replacing $w$ by $w v y$ in the both sides of (22), we have $G(x, w v y)=\sum_{n=0}^{\infty} a_{n}\left(Y_{n+r}^{\alpha-n k}(x ; k) y^{n}\right)(w v)^{n}$.

Operating $e^{w R}$ on both sides of (23), we get

$$
\begin{gathered}
e^{w R}(G(x, w v y)) \\
=e^{w R}\left(\sum_{n=0}^{\infty} a_{n}\left(Y_{n+r}^{\alpha-n k}(x ; k) y^{n}\right)(w v)^{n}\right) .
\end{gathered}
$$

Now the left member of (24), with the help of (8), reduces to

$$
\begin{gathered}
(1+k w y)^{\frac{1+\alpha-k}{k}} \exp \left(x-x(1+k w y)^{\frac{1}{k}}\right) G(x(1+ \\
\left.k w y)^{\frac{1}{k}}, \frac{w v y}{1+k w y}\right) .
\end{gathered}
$$

The right member of (24), with the help of (7), becomes $=\sum_{n=0}^{\infty} \sum_{p=0}^{n} a_{n-p} \frac{w^{p}}{p !} k^{p}(n+r-p+1)_{p} Y_{n+r}^{\alpha-n k}(x ; k) y^{n}(w v)^{n-p}$.
Now equating (25) and (26) and then substituting $w y=t$, we get

$$
\begin{gathered}
(1+k t)^{\frac{1+\alpha-k}{k}} \exp \left(x-x(1+k t)^{\frac{1}{k}}\right) G\left(x(1+k t)^{\frac{1}{k}}, \frac{v t}{1+k t}\right) \\
=\sum_{n=0}^{\infty} Y_{n+r}^{\alpha-n k}(x ; k) \sigma_{n}(v) t^{n}
\end{gathered}
$$

where

$$
\sigma_{n}(v)=\sum_{p=0}^{n} a_{p} k^{n-p}\left(\begin{array}{l}
n+r \\
p+r
\end{array}\right) v^{p}
$$

This completes the proof the theorem.

Special case 3: Now putting $k=1$ in our Theorem 1 we get the following result on generalised Laguerre polynomials:

Theorem 3. If there exists a unilateral generating relation of the form

$$
G(x, w)=\sum_{n=0}^{\infty} a_{n} L_{n+r}^{(\alpha-n)}(\mathrm{x}) w^{n}
$$

then

$$
\begin{gathered}
(1+t)^{\alpha} \exp (-x t) G\left(x(1+t), \frac{v t}{1+t}\right)= \\
\sum_{n=0}^{\infty} L_{n+r}^{(\alpha-n)}(\mathrm{x}) \sigma_{n}(v) t^{n},
\end{gathered}
$$

where

$$
\sigma_{n}(v)=\sum_{p=0}^{n} a_{p}\left(\begin{array}{l}
n+r \\
p+r
\end{array}\right) v^{p}
$$

which is found derived in [18].

Corollary 4: Putting $r=0$ in Theorem 3, we get the theorem found derived in $[18,19]$.

\section{Proof of Theorem 2}

$$
\begin{gathered}
\text { R.H.S. }=\sum_{n=0}^{\infty} \sigma_{n}(x, v) t^{n} \\
=\sum_{p=0}^{\infty} a_{p}(v t)^{p} \sum_{n=0}^{\infty}\left(\begin{array}{c}
p+r+n \\
n
\end{array}\right) Y_{p+r+n}^{\alpha-n k}(x ; k)(k t)^{n} \\
=\sum_{p=0}^{\infty} a_{p}(v t)^{p}(1+k t)^{\frac{1+\alpha-k}{k}} \exp \left\{x-x(1+k t)^{\frac{1}{k}}\right\} Y_{p+r}^{\alpha}\left(x(1+k t)^{\frac{1}{k}} ; k\right) \quad \text { [using (12)] } \\
=(1+k t)^{\frac{1+\alpha-k}{k}} \exp \left\{x-x(1+k t)^{\frac{1}{k}}\right\} \sum_{p=0}^{\infty} a_{p} Y_{p+r}^{\alpha}\left(x(1+k t)^{\frac{1}{k} ; k}\right)(v t)^{p} \quad \text { [using (3)] } \\
=(1+k t)^{\frac{1+\alpha-k}{k}} \exp \left\{x-x(1+k t)^{\frac{1}{k}}\right\} G\left(x(1+k t)^{\left.\frac{1}{k}, v t\right)}\right. \\
=L . H . S .,
\end{gathered}
$$

which is Theorem 2 . 
Corollary 5: If we put $r=0$ in Theorem 2, then we get the following Theorem:

Theorem 4. If

$$
G(x, w)=\sum_{n=0}^{\infty} a_{n} Y_{n}^{\alpha}(\mathrm{x} ; \mathrm{k}) w^{n}
$$

then

$$
\begin{gathered}
(1+k t)^{\frac{1+\alpha-k}{k}} \exp \left\{x-x(1+k t)^{\frac{1}{k}}\right\} G\left(x(1+k t)^{\frac{1}{k},} v t\right) \\
=\sum_{n=0}^{\infty} \sigma_{n}(x, v) t^{n}
\end{gathered}
$$

where

$$
\sigma_{n}(x, v)=\sum_{p=0}^{n} a_{p} k^{n-p}\left(\begin{array}{l}
n \\
p
\end{array}\right) Y_{n}^{\alpha-n k+p k}(\mathrm{x} ; \mathrm{k}) v^{p} .
$$

Special case 4. Now putting $k=1$ in our Theorem 2 and Theorem 4, we get the following results on generalised Laguerre polynomials:

Theorem 5. If there exists a unilateral generating relation of the form

$$
G(x, w)=\sum_{n=0}^{\infty} a_{n} L_{n+r}^{(\alpha)}(\mathrm{x}) w^{n}
$$

then

\section{Applications}

A1. As an application of our Theorem 1, we consider the following generating relation [11, 12]:

$$
\begin{gathered}
\sum_{n=0}^{\infty}\left(\begin{array}{c}
n+m \\
n
\end{array}\right) Y_{n+m}^{\alpha-n k}(x ; k) t^{n} \\
=(1+t)^{\frac{1+\alpha-k}{k}} \exp \left\{x-x(1+t)^{\frac{1}{k}}\right\} Y_{m}^{\alpha}\left(x(1+t)^{\frac{1}{k}} ; k\right) .
\end{gathered}
$$

If in our theorem, we take $a_{n}=\left(\begin{array}{c}n+m \\ n\end{array}\right)$, then

$$
\begin{gathered}
G(x, w) \\
=(1+w)^{\frac{1+\alpha-k}{k}} \exp \left\{x-x(1+w)^{\frac{1}{k}}\right\} Y_{m}^{\alpha}\left(x(1+w)^{\frac{1}{k}} ; k\right) .
\end{gathered}
$$

Therefore by the application of our Theorem 1, we get the following generalization of the result (36):

$$
\begin{aligned}
& (1+k t+v t)^{\frac{1+\alpha-k}{k}} \exp \left\{x-x(1+k t+v t)^{\frac{1}{k}}\right\} Y_{m}^{\alpha}\left(x(1+k t+v t)^{\frac{1}{k} ; k}\right) \\
& =\sum_{n=0}^{\infty} Y_{n+m}^{\alpha-n k}(x ; k) \sigma_{n}(v) t^{n},
\end{aligned}
$$

where

$$
\sigma_{n}(v)=\sum_{p=0}^{n} a_{p} k^{n-p}\left(\begin{array}{c}
n+r \\
p+r
\end{array}\right) v^{p} .
$$

A2. As an application of Theorem 2, we consider the following generating relation $[9,11,12]$ :

$$
\begin{gathered}
\sum_{n=0}^{\infty}\left(\begin{array}{c}
n+r \\
n
\end{array}\right) Y_{n+r}^{\alpha}(x ; k) t^{n} \\
=(1-t)^{\frac{-1-\alpha-r k}{k}} \exp \left\{x-x(1-t)^{-\frac{1}{k}}\right\} \times
\end{gathered}
$$




$$
\times Y_{r}^{\alpha}\left(x(1-t)^{\left.-\frac{1}{k} ; k\right)}\right.
$$

If in our theorem, we take $a_{n}=\left(\begin{array}{c}n+r \\ n\end{array}\right)$, then

$$
G(x, w)=(1-w)^{\frac{-1-\alpha-r k}{k}} \exp \left\{x-x(1-w)^{-\frac{1}{k}}\right\} Y_{r}^{\alpha}\left(x(1-w)^{-\frac{1}{k}} ; k\right)
$$

Therefore by the application of our Theorem 2 we get the following generalization of (38):

$$
\begin{aligned}
& (1+k t)^{\frac{1+\alpha-k}{k}}(1-v t)^{\frac{-1-\alpha-r k}{k}} \exp \left\{x-x(1+k t)^{\frac{1}{k}}(1-v t)^{-\frac{1}{k}}\right\} \\
& \times Y_{r}^{\alpha}\left(x(1+k t)^{\frac{1}{k}}(1-v t)^{\left.-\frac{1}{k} ; k\right)}\right. \\
& =\sum_{n=0}^{\infty} t^{n} \sum_{p=0}^{n} a_{p} k^{n-p}\left(\begin{array}{c}
n+r \\
p+r
\end{array}\right) Y_{n+r}^{\alpha-n k+p k}(\mathrm{x} ; \mathrm{k}) v^{p} .
\end{aligned}
$$

For $k=1$, we get the corresponding generalization of the result involving generalized Laguerre polynomials, $L_{n}^{(\alpha)}(x)$.

\section{Conclusions}

From the above analysis, it is clear that whenever one knows a generating relations of the form $(1,3)$ then the corresponding bilateral generating relations can at once be written down from $(2,4)$. So one can get a large number of bilateral generating relations by attributing different suitable values to $a_{n}$ in $(1,3)$.

\section{Acknowledgements}

The authors are greatly indebted to Prof. A.K. Chongdar of IIEST, Shibpur for his constant encouragement and guidance.

\section{REFERENCES}

[1] Konhauser, J. D. E. , Some properties of biorthogonal polynomials, J. Math. Anal. Appl., 11 (1965), 242-260.

[2] Konhauser, J. D. E. , Biorthogonal polynomials suggested by the Laguerre polynomials, PacificJ. Math., 21 (1967), 303-314.

[3] Spencer, L. and Fano, U., Penetration and diffusion of X-rays, calculation of spatial distribution by polynomial expansion, J. Res. Nat. Bur. Standards 46(1951), 446-461.

[4] Erdelyi, Arthur, with Magnus, W., Oberhettinger, F., Tricomi, F.G., et al.,Higher transcendental function, vol. 2. New York: McGraw-Hill 1953.

[5] Truesdell, C., An essay toward a unified theory of special functions, Princeton university press, 1948.

[6] McBride, E.B.:Obtaining Generating Functions, Springer Verlag, Berlin, 1972.

[7] Carlitz, L., A note on certain biorthogonal polynomials, Pacific J. Math., 24 (1968), 425-430.

[8] Prabhakar, T.R., On a set of polynomials suggested by the
Laguerre polynomials, Pacific J. Math., 35 (1970), 213-219.

[9] Prabhakar, T.R., On the other set of biorthogonal polynomials suggested by Laguerre polynomials, Pacific J. Math., 37 (1971), 801-804.

[10] Patil, K.R. and Thakare, N.K., Multilinear generating function for the Konhauser biorthogonal polynomial sets,SIAM J. Math. Anal., 9(1978), 921-923.

[11] Srivastava, H.M., Some Biorthogonal polynomials suggested by the Laguerre polynomials, Pacific J. Math., 98(1) (1982), $235-250$

[12] Srivastava, H.M., A note on the Konhauser sets of Biorthogonal polynomials suggested by the Laguerre polynomials, Pacific J. Math., 90(1) (1980), 197-200.

[13] Srivastava, A.N. and Singh, S.N., On the Konhauser polynomials $Y_{n}^{\alpha}(x ; k)$, Indian J. pure appl. Math., 10(1979), $1121-1126$

[14] Shreshtha, R.M. and Bajracharya, S., Group theoretical study of a bilateral generating function, Int. Trans. and Spec. func., 5(1-2) (1997), 147-152.

[15] Carlitz, L., A note on the Laguerre polynomials, MichiganMath. J.,7(3) (1960), 219-223.

[16] Al-Salam, W.A., Operational representations for the Laguerre and other polynomials, Duke Math. Jour., 31(1964),127-142.

[17] Calvez, L.C. et Genin, R., Applications des relations entre les Fonctions génératrices et les formules de type Rodrigues, C.R. Acad. Sci. Paris Ser. A-B, 270(1970), A41-A44.

[18] Alam, S. and Chongdar, A.K., On generating functions of modified Laguerre polynomials, Rev. Real Academia de Ciencias Zaragoza, 62(2007), 91-98.

[19] Das,S. and Chatterjea, S.K., On a partial differential operator for Laguerre polynomials, Pure Math. Manuscript, 4(1985), 187-193.

[20] Chongdar, A.K., Some generating functions involving Laguerre polynomials, Bull. Cal. Math. Soc., 76(1984), 262-269.

[21] Majumder, A.B., Some generating functions of Laguerre polynomials, J. Ramanujan Math. Soc., 10(2)( 1995), 
195-199.

[22] Desale, B.S. and Qashash, G.A., A general class of generating functions of Laguerre polynomials, Jour. Ineq. Special fun., 2(2011), 1-7.
[23] Chongdar, A.K., Pittaluga, G. and Sacripante, L., On generating functions for certain special functions by Weisner's group theoretic method, Math. Bal., 12(1998),new series, 369-381. 